\title{
Sympathetic input into the enteric nervous system
}

\author{
O Lundgren
}

\section{Introduction}

The basic concepts of the autonomic nervous system of organs and tissues were formulated around the turn of the century and summarised in a classical monograph by Langley (1921). ${ }^{1}$ The detailed distribution of the sympathetic nerves was, however, not elucidated until it became possible to stain sympathetic neurones specifically using the FalckHillarp technique. When this technique was applied to the gastrointestinal tract the picture seen was in some respects surprising. At the time it was generally believed that most organs and tissues had a dual innervation (sympathetic and parasympathetic) with opposite effects on function. It seemed, therefore, puzzling when the Falck-Hillarp technique revealed that innervation of a major part of the gastrointestinal wall, the smooth muscle layers, was very scarce. Instead it was found that most adrenergic fibres make contact with one of the two major plexuses in the alimentary canal. Adrenergic innervation is seen in the mucosa, in particular around the crypt epithelium. ${ }^{2}$ The functional consequences of this distribution are discussed below.

This brief review of the interplay between the sympathetic and enteric nervous systems will discuss the control of blood vessels, epithelial transport, motility, and endocrine cells (the enterochromaffin cells in particular).

\section{Gothenburg \\ University, \\ Medicinaregatan 11, 40033 Gothenburg, \\ Sweden \\ O Lundgren}

Dhysiology,

Corresponding author: Professor O Lundgren. ove.lundgren@fysiologi.gu.se nerves to the gut elicits a blood flow response which is very characteristic. Immediately on electrically stimulating the splanchnic nerves, pronounced vasoconstriction is observed which, however, subsides within a few minutes to reach a steady state level of blood flow. It seems probable that flow during steady state represents the "physiological" response to nerve activation. Intestinal flow resistance is thus only moderately increased (2-3 times) even when stimulating the sympathetic vasoconstrictor nerves at high rates $(8-16 \mathrm{~Hz})$. When investigating blood flow distribution within the intestinal wall it has been demonstrated that villus blood flow is not under neural influence during the steady state part of vasoconstriction. The decrease in blood flow occurs in the crypts and muscle layers. ${ }^{3}$

The sympathetic nerves not only control resistance vessels; stimulation frequency dependent constriction of the veins is observed, decreasing regional blood volume by $40 \%$ at most. Furthermore, the capillary filtration coefficient is markedly decreased, which indicates that the number of perfused capillaries is attenuated, presumably secondary to constriction of precapillary sphincters.

The enteric nervous system (ENS) also controls intestinal blood flow. In vivo studies have shown that mechanical stimulation of the intestinal mucosa evokes marked vasodilatation. This response is apparently neurally mediated as it is abolished by nerve blocking agents (tetrodotoxin or lidocaine). The vasodilatation cannot be blocked by cholinergic receptor blockers, suggesting that there is no cholinergic synapse in the reflex. Furthermore, the response is not influenced by "chronic" denervation of the intestine, implying that vasodilatation is not mediated via an axon reflex of the "classical" type. However, 5-hydroxytryptamine (5-HT) tachyphylaxis or serotonin receptor blockers attenuate the vasodilatation caused by mucosal mechanical stimulation. Based on such observations it was suggested that mechanical stimulation causes release of 5-HT from enterochromaffin (EC) cells which in turn stimulate dendrites located just underneath the intestinal epithelium. ${ }^{4}$ This is illustrated schematically in fig 1A. It should be emphasised that the detailed arrangement of this vasodilator reflex is not known. Some observations made by Surprenant ${ }^{5}$ suggest the possibility that an axon reflex may be involved.

In contrast with sympathetic control of motility and epithelial transport, vasodilatation evoked by mechanical mucosal stimulation is not influenced by stimulating sympathetic

Abbreviations used in this paper: $5-\mathrm{HT}$,

5-hydroxytryptamine; EC, enterochromaffin; CNS, central nervous system; ENS, enteric nervous system. 
vasoconstrictor fibres. ${ }^{4}$ The nervous arrangement of the local vasodilator mechanism is apparently organised in a different way from that of motility and fluid transport.

Fluid and electrolyte transport

The morphological observations of the adrenergic innervation briefly summarised in the introduction suggest two sites of action for the control of epithelial transport: a direct action on epithelial cells and an indirect action on enteric neurones, in particular those of the submucous plexus. Evidence for the latter mechanism has been obtained in electrophysiological studies which have shown that the non-cholinergic submucous neurones, presumably controlling epithelial functions, are under adrenergic influence via inhibitory postsynapatic potentials. ${ }^{6}$ This action evokes a decrease in electrolyte and fluid tissue to lumen transport, for example. Interestingly, cholinergic submucous neurones do not receive any such innervation. ${ }^{6}$

It is well established that many secretory states of the intestinal tract, including cholera, are evoked via activation of the ENS. Figure $1 \mathrm{~B}$ illustrates the simplest model for the nervous secretory reflex of the ENS stimulated by cholera toxin that can be constructed from our current knowledge. There is experimental evidence for the view that cholera toxin and some bile salts, at least in part, activate the ENS via release of 5-HT from EC cells. In line with the electrophysiological observation described above it has been shown that splanchnic nerve stimulation inhibits fluid secretion caused by cholera toxin. This effect is more pronounced the higher the rate of fluid secretion and is probably explained by a higher activity in the ENS when toxin induced fluid secretion is large. Hence the sympathetic inhibitory influence is dependent on the level of activity in the secretory nervous reflexes of the ENS (fig 1B). ${ }^{7}$ There is also experimental evidence for a direct action of neurally released noradrenaline on crypt enterocytes although this effect is less important than the nervous effect on the ENS.

Sympathetic nervous control of epithelial fluid transport in the small intestine can be reflexly activated by deloading baroreceptors in the aorta and neck arteries and/or intrathoracic volume receptors. Thus sympathetic nerves are part of the homeostatic system controlling fluid and electrolyte content of the body. ${ }^{8}$

\section{Motility}

As noted in the introduction, the Falck-Hillarp technique showed that few adrenergic fibres reached the muscle layers of the gastrointestinal tract. This implies that sympathetic control of the smooth muscle layers is to a large extent not a direct but an indirect action, except for the direct effect of blood borne catecholamines released from the adrenal glands. There is experimental evidence for adrenergic inhibition of local excitatory motor reflexes and/or extrinsic excitatory parasympathetic nervous activity. Hence the magnitude of the sympathetic effect is dependent on ongoing activity in, for example, the vagal excitatory nerves to the
Enterochromaffin cell

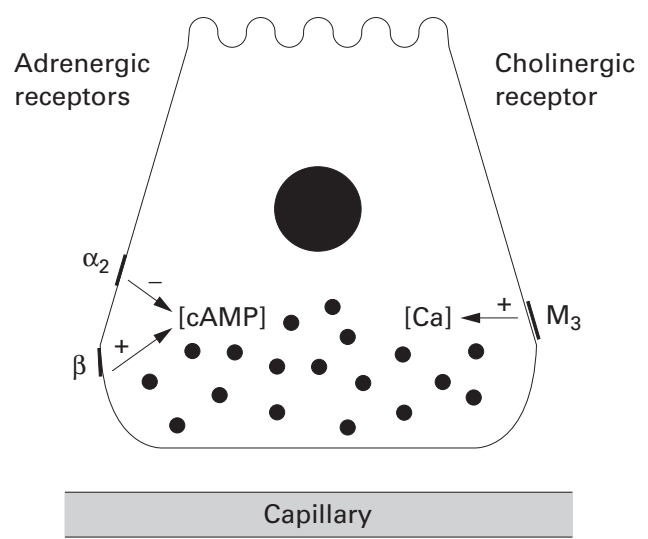

Figure 2 Adrenergic and cholinergic control of the release of 5-hydroxytryptamine (5-HT) from enterochromaffin cells. cAMP, cyclic adenosine monophosphate.

smooth muscle layers in a manner similar to that described for sympathetic control of fluid and electrolyte transport (fig 1C). ${ }^{9}$

It is apparent from figure 1 that sympathetic nervous control of epithelial transport and motility is organised in a similar way, namely control is exerted at a synaptic site. The sympathetic nerve may exert its action at a synapse in at least two ways. It may inhibit the release of acetylcholine, for example, from an intramural synapse, and/or noradrenaline released from sympathetic nerves may hyperpolarise the membrane potential of the postsynaptic neurone. There is experimental evidence for both mechanisms. ENS control of blood flow, lacking at least a cholinergic synapse, is not influenced by sympathetic neurones.

\section{Enterochromaffin cells}

The gastrointestinal tract is the largest endocrine organ in the body, containing a large number of peptides/amines located in endocrine cells dispersed among the transporting epithelial cells. It is well established that different chemicals in the intestinal lumen can evoke the release of specific peptides. Hence glucose causes release of gastric inhibitory peptide, and long chain fatty acids evoke release of cholecystokinin and neurotensin. Endocrine cells may also be sensitive to luminal physical variables. EC cells have been proposed as mechanoreceptors, releasing 5-HT on distortion of the villi.

Based on studies performed mainly in EC cells, it seems probable that release of hormones from endocrine cells of the gastrointestinal tract are under control of the autonomic nervous system. Figure 2 summarises, in a schematic manner, the adrenergic and cholinergic control of release of 5-HT from EC cells. There are both $\alpha$ and $\beta$ adrenergic receptors on EC cells. Release studies performed in vivo indicate that $\alpha$ adrenergic agonists inhibit release of 5-HT from cells whereas the cholinergic influence has the opposite effect. ${ }^{10}$ As suggested in figure 2 , these effects may be mediated via changes in intracellular cyclic adenosine monophosphate and/or $\mathrm{Ca}^{2+}$ concentrations. 
Autonomic nervous control of endocrine cells of the gastrointestinal tract may have interesting functional implications. It is well established that the sensitivity of the muscle spindles in skeletal muscle is controlled from the central nervous system (CNS) via efferent fibres influencing the so called intrafusal muscle cells. One can envisage similar CNS control of the sensitivity of endocrine cells of the gastrointestinal epithelium via the receptors illustrated in fig 2 . Activation of these receptors may change the sensitivity of endocrine cells to luminal stimuli by influencing membrane potential. Thus the sympathetic nervous system may indirectly influence the activity of ENS neurones via control of luminal sensory receptors. In the context of the present symposium, this may be particularly interesting for those endocrine cells which, via release of peptides/ amines, activate afferent neurones located adjacent to their basolateral membrane.
1 Langley JN. The autonomic nervous system. London: Heffner, 1921.

2 Norberg K-A. Adrenergic innervation of the intestinal wall studied by fluorescence microscopy. Int $\mathcal{F}$ Neuropharmacol 1964;3:379-82

3 Lundgren O. Microcirculation of the gastrointestinal tract and pancreas. In: Renkin EM, Michel CC, eds. Handbook of physiology: The cardiovascular system. Bethesda: American Physiological Society, 1984; IV:799-863.

4 Biber B. Vasodilator mechanisms in the small intestine. An experimental study in the cat. Acta Physiol Scand 1973;401: $1-31$.

5 Surprenant A. Control of the gastrointestinal tract by enteric neurons. Annu Rev Physiol 1994;56:117-40.

6 Bornstein JC, Furness JB. Correlated electrophysiological and histochemical studies of submucous neurons and their contribution to understanding enteric neural circuits. $\mathcal{F}$ Auton Nerv Syst 1988;25:1-13.

7 Sjövall H. Sympathetic control of jejunal fluid and Sjövall H. Sympathetic control of jejunal fluid and
electrolyte transport. Acta Physiol Scand 1984;(suppl 535): $1-62$

8 Sjövall H, Redfors S, Jodal M, et al. On the mode of action of the sympathetic fibres on intestinal fluid transport: Evidence for the existence of a glucose stimulated secretory nervous pathway in the intestinal wall. Acta Physiol Scand 1983;119:39-48

9 Jansson G, Martinson J. Studies on the ganglionic site of action of sympathetic outflow to the stomach. Acta Physiol Scand 1966;68:184-92.

10 Racké K, Schwörer H. Regulation of 5-HT release from enterochromaffin cells. Behav Brain Res 1996;73:83-7. 